\title{
SciDoc
}

Infer, Interpret \& Inspire Science

International Journal of Dentistry and Oral Science (IJDOS)

ISSN: $2377-8075$

\section{Solitary Plasmacytoma of the Oral Cavity with Review of Literature: A Case Report}

Case Report

\author{
Shivananda $\mathrm{S}^{1}$, Vidya G Doddawad ${ }^{2 *}$, Manjula $\mathrm{S}^{3}$, Aruna $\mathrm{G}^{4}$, M.S.Muni Sekhar ${ }^{5}$
}

${ }^{1}$ Associate Professor, Department of Oral And Maxillofacial Surgery, JSS Dental College and Hospital, A constituent college of JSSAHER, Mysore-570022, India.

${ }^{2}$ Associate Professor, Department Of Oral Pathology And Microbiology, JSS Dental College and Hospital, A constituent college of JSSAHER, Mysore-570022, India.

3 Associate Professor, Department of Oral And Maxillofacial Surgery, JSS Dental College and Hospital, A constituent college of JSSAHER, Mysore-570022, India.

${ }^{4}$ Senior Lecturer, Department of periodontics, JSS Dental College and Hospital, A constituent college of JSSAHER, Mysore-570022, India.

${ }^{5}$ Lecturer, Oral Pathology Disvision, Department of Preventive Dentistry, College of Dentistry, Jouf University, Sakaka, Aljouf, Kingdom of Saudi Arabia.

\section{Abstract}

Solitary bone plasmacytoma is considered as a3\% of all plasma cell containing tumors anduncommon condition in the head and neck region especially in the jaws which demonstrate as a single bone tumor.Based on clinical behavior and prognosis, multiple myeloma is differing from the solitary plasmacytoma. The incidence rate of localized plasmacytoma is lesser than the multiple myeloma.However, localised bone plasmacytoma is less commonly manifest among plasma cell tumour. Only $3 \%$ of Solitary bone plasmacytoma is a plasma cell tumor and considered as an immunoproliferative monoclonal disease. Localized plasmacytoma are rare in occurrence than multiple myeloma andappear as a single and extra osseous form of bone neoplasm. Solitary bone plasmacytoma seen in the long bones, vertebrae, sternum,clavicle, rib and humerus are the most common sites, but rarely involves maxillofacial region. Here, we report a rare case of solitary plasmacytoma affecting a mandible which is diagnosed based on the clinical, radiological, hematological, and histopathological findings.

Keywords: Lymphoproliferative Disorder; Mandible; Maxillofacial; Plasma Cell Tumor; Plasmacytoma; Solitary Bone.

\section{Introduction}

Plasma cell tumors are divided into solitary bone plasmacytoma (SBP), extramedullary plasmacytoma (EMP) and multiple myeloma (MM) which are B-cell lymphoid neoplasms [1]. The other rare variants of plasma cell tumors are non-secretory myeloma, indolentmyeloma, smoldering myeloma, plasma-cell leukemia (PCL). However, localised bone plasmacytoma is less commonly manifest among plasma cell tumour. Localized plasmacytoma are rare in occurrence than multiple myeloma andappear as a single and extra osseous form of bone neoplasmc [2]. Solitary bone plasmacytoma seen in the long bones, vertebrae, sternum,clavicle, rib and humerus are the most common sites, [3] but rarely involves maxillofacial region. $4.4 \%$ occur in the mandible, especially in the body of the mandible, angle and ramus of the mandible due to bone marrow-rich areas [3, 4]. The extramedullary plasmacytomas commonly seen on the mucosal surfaces such as nasal sinuses, oropharynx, larynx etc.[1] Few authors reported that $18 \%$ in the oropharynx, $20 \%$ inthe nasopharynx, and $40 \%$ occur in the nasal cavity and paranasal sinus [2]. Therefore, both two diseases are recognized as two separate entities [5].

Only 3\% of Solitary bone plasmacytoma is a plasma cell tumor and considered as an immunoproliferative monoclonal disease [2]. Monotypic cytoplasmic immunoglobulins and plasma cell-associated antigens, with an absence of immature B-cell antigens will be enlisted as atypical plasma cells [5]. Solitarybone plasmacytoma may be alocalized disease and sometime, it may be considered as the first early manifestation of a multiple myeloma. The biological behavior of these tumors is variable from localized form

*Corresponding Author:

Dr. Vidya Gowdappa Doddawad,

Associate Professor, Department Of Oral Pathology And Microbiology, JSS Dental College and Hospital, A Constituent College of JSSAHER, Mysore-570022, India. Tel: 09343830621

E-mail: drvidyagd@gmail.com

Received: March 22, 2021

Accepted: August 30, 2021

Published: September 05, 2021

Citation: Shivananda S, Vidya G Doddawad, Manjula S, Aruna G, M.S. Muni Sekhar. Solitary Plasmacytoma of the Oral Cavity with Review of Literature: A Case Report. Int J Dentistry Oral Sci. 2021;8(9):4337-4340. doi: http://dx.doi.org/10.19070/2377-8075-21000882

Copyright: Dr.Vidya Gowdappa Doddawad ${ }^{\circ} 2021$. This is an open-access article distributed under the terms of the Creative Commons Attribution License, which permits unrestricted use, distribution and reproduction in any medium, provided the original author and source are credited. 
(SBP and EMP) to MM in the clinical like latency to rapid growth and progression. The solitary bone plasmacytoma have a better prognosis, while compare to multiple myeloma which may be different. Durie and Salmonstaging system on plasma cell tumor enumerated that plasmacytoma as a stage I multiple myeloma 6 and even many researchers were also opined that solitary bone plasmacytoma as an early manifestation of multiple myeloma [2]. Maxilla/mandible is a rare occurrence of SBP and clinical presentations are less compared to multiple myeloma, hence it will be underestimated to diagnoseand considered as nonspecific. Therefore, we are planning to report a rare case of mandibular solitary plasmacytoma which was diagnosed based onclinical, radiograph and histopathology findings.

\section{Case Report}

A 38-year-old female patient reported to a clinic, with a complaint of pain in the right lower mandible region since 6months. The pain was intermittent in nature but sudden in onset was noticed since last two weeks. (Fig. 1) The medical history and physical examination of the patient revealed no abnormality detected. Intra oral examination exhibits the presence of caries roots in relation to 48 and buccal vestibule was obliterated. The right submandibular lymph nodes were noticeable, measuring about $1.5 \mathrm{X} 1 \mathrm{~cm}$ in dimension, firm in consistency, non-tender on palpation and not fixed to the underlying tissue.

Orthopantomography and Intraoral periapical radiograph revealed carious root stumps and extensive solitary, round, unilocular radiolucent lesion with sclerotic border in relation to 48 regions. The lesion shows buccal cortical bone expansion can be appreciated. (Fig. 2\&3) Hematological investigation explained about there was anelevated leukocytic count and near to normal hemoglobin levels. The alkaline phosphatase levels were within normal range but serum calcium levels expressed a little change, and Hepatitis B and HIV results were negative. On urine examination, it was rule out the multiple myeloma by Bence-jones protein which showed negative.

Incisional biopsy was done for histopathological examination and it showed highly cellular inflammatory infiltrate which is sheets of malignant plasma cells with a diffuse, monotonous and variably differentiated. (Fig. 4) The nucleus of malignant cells was large, round/oval in shape with vesicular nuclei and prominent nucleoli. (Fig. 5) Immunohistochemical studies revealed that section as positive for CD138 was positive but slide and slide blocks were not procured since it was taken by patient.

Figure 1. Clinical picture of female patient having alesion in the right lower jaw region.

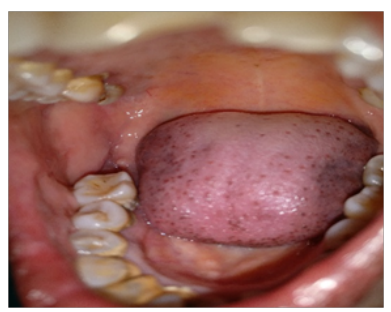

Figure 2. Orthopantomograph shows carious root stumps and extensive solitary, round, unilocular radiolucent lesion with sclerotic border.

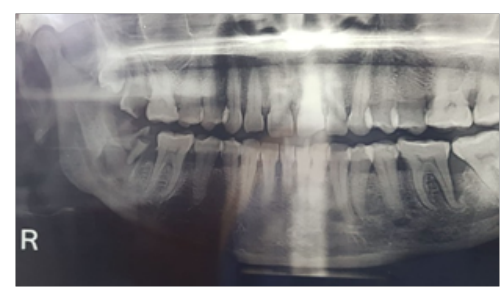

Figure 3. Intraoral periapical radiographshows carious root stumps with unilocular radiolucent lesion.

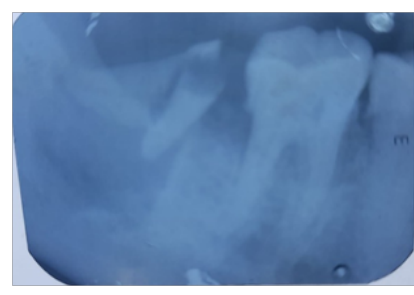

Figure 4. H\&E section showing dense cellular infiltrate, which is diffuse, sheets of malignant plasma cellsalong with the surface epithelium. (100X)

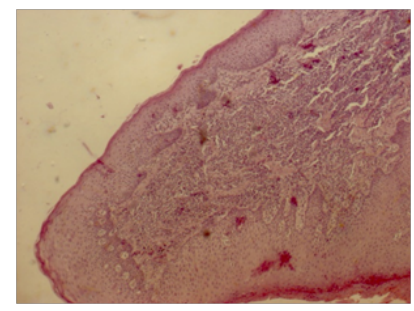


Figure 5. H\&E section showing the malignant plasma cells which is large, round/oval shape with vesicular nuclei. (400X).

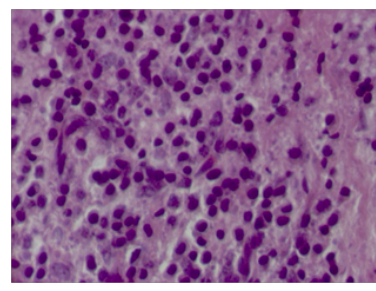

Based on the clinical, radiological, hematological, histopathology and immunohistochemical findings, the final diagnosis of solitary bone plasmacytosis was made. The patient was referred to higher cancercenter for complete treatment. The treatment of SBP includes radiation therapy,chemotherapy, radical surgery, or a combination.

\section{Discussion}

Schridde in 1905, first described about plasmacytoma is anuncommon solitary growth of neoplastic monoclonal plasma cells [2]. Plasma cell myeloma is a multicentric in origin in which monoclonal neoplastic proliferation of plasma cells of bone-marrowderivation. In due course of time, cells get infiltrates in various organs of the body but it mayproduce plasma cell leukemia. It is basically osteolytic lesions which manifest bone pain, hypercalcemia, a monoclonal gammopathy, and seen a deposition of abnormal immunoglobulin chains (amyloid) in various organs like long bone, kidney $[2,3]$. The incidence rate of plasma cell tumor is about 2.6 to 3.3/lac population in the world but higher in African- American origin people [1]. The long term inhaled irritant or viral infection may be the main aetiology. Later, based on cytogenetic studies,it was shown the characteristic numeric abnormalities seen in plasma cell neoplasms are losses in chromosome $13,14,8,14$, and $X$, and gains of chromosome 1,3, 5, 7, 9, 11, 15, and 19. Translocations involving theimmunoglobulin heavy chain $(\mathrm{IgH})$ locus occur at high frequency (50\%-70\% of cases). In $40 \%$ of these cases, the translocation partner is one of five loci: $11 \mathrm{q} 13$ (bcl-1/cyclin D1 locus), 6p21 (cyclin D3), 4p16 (fibroblast growth factor receptor 3 and multiple myeloma SET domain), $16 \mathrm{q} 23$ (c$\mathrm{MAF}$ ), and 20q11 (MAFB) 2. The principal growth factor in the progression of plasma cell disorders is IL-6. The SBP was observed and thought that itcould be an early stage in the progression from monoclonal gammopathy to multiple myeloma.[7]

Plasma cell neoplasms usually affect older people age about 50-80 yearswith a male: female ratio of 3:1. Canger et.alnoted slight male predilection with 2:1 male to female ratio.[2] Clinical manifestations may be performpoor and difficult to diagnose as solitary plasma cytoma of jaw. Specific clinical features of solitary plasmacytoma are unique such as pain and bone swelling, loss of bone function and movements or pathological fractureswith or without bone erosion. [3] The present case shows female patient about 32 yrs of age and complains of carious root stumps associated with pain.

Bone imaging is aappropriate investigation fordiagnosing solitary plasmacytoma which includes plainradiographs, computed tomography (CT) scan,magnetic resonance imaging (MRI), and PET scan. Routine radiographs are less expensive than other scan, but it shown to possess greater ability to detectcortical bone condition and associated lesion than other scan.[4] On radiographic images, it appearas unilocular or multilocularradiolucent destructive lesions, [2] and without any reactive bone formation. [3] Plasma cells which stimulate the growth of osteoclasts with the help of osteoclast activating factors, resulting in bone resorption. The present case, radiograph shows expansile unilocular, round radiolucency at the left ramus of the mandible with buccal cortical plate expansion.

Histopathologically, these type of tumors shows sheets of plasma cells with avarying degree of differentiation of atypical plasma cells i.e.abundant cytoplasm and eccentrically located nuclei. There will be an inclusion bodies and cart wheel chromatin patternseen. [3] All the above feature was consistent with the present case.

IHC markers such as LCA, EMA, CD45 and CD138 are essential to show abnormal hematopoietic activity. CD138 is a marker for plasma cells, plasmablasts, and some immunoblasts. Its main reactivity in hematolymphoid neoplasms includes plasma cell neoplasms and some large B-cell lymphomas. CD 45 is specific for both benign and malignant lymphoid (both B-and T-) cells. LCA does not stain megakaryocytes, erythroid cells, normal myeloid cells, or nonhematopoietic cells. EMA is an excellent marker for most normal and neoplastic epithelia but is also expressed by mesotheliomas, meningiomas, and mesenchymal neoplasms. [8]

Plasma cell has both kappa or lambda immunoglobulin lightchain are demonstrated by IHC (immunohistochemistry)in inflammation because plasma cells are polyclonal. But SBP has either kappa orlambda light chain, since atypical plasma cells are monoclonal in nature. The monoclonal SBP of IHC reactivity showed only positive for thelambda light chain, hence it is easily differentiated SBP from an inflammatory condition. Therefore, CD138 positivity is a basic criterion helped to arrive at a confirmatory diagnosis ofsolitary bone plasmacytoma.[2]

The other laboratoryinvestigations of solitary plasmacytoma are immunoglobulin production,serum $\beta$-2-microglobulin levels, kidney dysfunction and blood calcium alterations. [3] Systemic alterations seen in multiple myeloma such as hypercalcemia, dysfunction of kidney, bone lesions, anemia, leukopenia,thrombocytopenia, and proliferation of monoclonal plasma cells in the bone marrow. Therefore, Ig production likebeta 2-microglobulin analysis, complete blood cell count (CBC), serum calcium,serum phosphorous, $\mathrm{C}$ reactive protein (CRP), and skeletal bone surveys are highlyrecommended.[2]

Differential diagnoses of SBP may include metastatic lesion, Osteoid osteoma, Osteoblastoma, Ewings sarcomas, Rhadomyosarcomas, and Histocytomas. Metastatic lesions are osteolytic in nature which shows localized radio lucency that resembles SBP. Osteoidosteoma is a solitary benign tumor which bone in origin, 
thataffects long bone cortices. It clinically manifests with history of nocturnal pain and immediatelyrelieved by non-steroidal antiinflammatory drugs. Osteoblastoma is a rare benignosseous tumor, present with a painfulbony swelling. This tumor explains the bone formation in the presence of abundant osteoblasts. [4] Histocytomas are usuallyseen in younger individuals andthere were no reports are available regarding the presence of any primary lesion.[1] Therefore, SBP is diagnosed based on clinical history, and other investigations such as imaging techniques, biopsy, and special stains (IHC) which are helpful in differentiating SBP from other lesions. $[4$

The treatment options are radiation, chemotherapy and surgery. The literature stated that combination of radiation and surgical routeswill have agood result with good prognosis and less local recurrence.[3] Solitary plasmacytomas are highly radiosensitive lesions.1The Radical radiotherapy dose is 40-50 Gy and the best way of treatment of choice with a $80 \%$ recovery rate. Surgical treatmentwill be done to remove the entire tumor with minimal esthetic problem, and to prevent \& stabilize a bone by pathologic fracture. The role of chemotherapy is not clearly understood. Currently, the research is going on the role of angiogenesis inhibitors, thalidomide, proteaseinhibitors or inhibitors of vascular endothelium growth factor in plasma cell neoplasms. This chemotherapy may take primary alternative treatment in future. [2] The combination of chemotherapy and radiotherapyhas not been shown any prognosisas compared to local treatment with radiotherapy alone for the treatment of SBP. But,for few cases, additional chemotherapy should be provided for those cases progressing tomultiple myeloma.[2]

The solitary bone plasmacytoma is a benign tumor and manifest as osteolytic lesion with better prognosis. Nearly $80 \%$ of SBP patients do not show any recurrence even after 5 years and 50$80 \%$ patients have 10 year survival rate of SBP. [1, 4] Few authors believed that the SBP is afirst stage of the MM because 35\% - $85 \%$ of SBP cases will develop into MM in few months toyears after diagnosis. The chances of SBP transform MM depending upon few risk factors like age ( $>60$ years), Mcomponent levels $>20$ $\mathrm{g} / \mathrm{L}$ up to one year following radiotherapy, size of the tumour $(>5 \mathrm{~cm})$ and vascularity. So, after the treatment of SPB cases itis compulsory to do closely followed up.[2]

Solitary bone plasmacytoma manifests itself as a single benign osteolytic lesion with better prognosis than multiple myeloma. [1, 9] Patients showed better prognosis in few cases like age $<60 \mathrm{yr}$ had significantly better survival rate $(90 \%$ for patients aged $0-29 \mathrm{yrs} \&$ $80 \%$ for patients $30-59 \mathrm{yrs}$.) when compared to patients diagnosed at age $>60 \mathrm{yrs}$ ( 5 yr. survival $45 \%$ ). [1] Long-term follow up should be carried out to detect recurrence or manifestation of MM.[10]

Therefore, the basic criteriato diagnosis a solitary plasmacytoma was given below.[2]

- Isolated area of bone destruction due to clonal plasma cells

- Bone marrow plasma cell infiltration not exceeding 5\% of all nucleated cells

- Absence of further osteolytic bone lesions or other tissue involvement (ie, no evidenceof systemic plasmacytoma)

- Absence of anemia

- No hypercalcemia, or renal impairment

- Absence of any changes in immunoglobulin chemistry orlow concentrations of serum or urine monoclonal protein (i.e., myeloma protein), or preserved levels of immunoglobulins.

Finally, SBP is a rare benign tumor of the head and neck region that constitutes approximately $3 \%-10 \%$ of all plasmacell neoplasms. The clinical features of SBP may be less common when compare to MM, thus it is considered as early stage of MM. Therefore, an early diagnosis and treatment of SBP may be challenge to all the surgeons to perform.[2] The clinical and radiographic findings of SBP may not be idealistic, butclinician should alwaysremember to considerasone of the differential diagnosis in the oral and maxillofacial lesions.[3]

\section{Conclusion}

Solitary bone plasmacytoma classified as lympho proliferative clinical disorders which consists of clonalproliferation of plasma cells. Solitary plasmacytoma typically seen in a single bone or extramedullary site andusually seen in the vertebrae or thorax, but rarely involves the mandible. Therefore,knowledge of the solitary bone plasmacytoma in the maxillofacial region is important for the oral surgeon to recognize at an early stage and optimal treatment canbe rendered to these patients before it arrives at complication.

\section{References}

[1]. Kamal M, Kaur P, Gupta R, Gupta S, Singh S. Mandibular plasmacytoma of jaw-a case report. Journal of clinical and diagnostic research: JCDR. 2014 Aug;8(8):ZD20.

[2]. FaseelaBeegum, Sudha sivasankar, Reshmi G Nair,Shijineed T K,Hasna K K. Unusual Presentation of Solitary Plasmacytoma of Mandible withReview of Literature. Journal of Dental and Medical Sciences 2017;16(5): 67-72.

[3]. Singh A, Singh V, Sharma N. Solitary Plasmacytoma of Mandible: A rare case report.

[4]. Baad R, Kapse SC, Rathod N, Sonawane K, Thete SG, Kumar MN. Solitary plasmacytoma of the mandible - a rare entity. J Int Oral Health. 2013 Jun;5(3):97-101. Pubmed PMID: 24155610.

[5]. Balreddy P, Prashanth Panta, Sridhar Reddy, Sandhya Gokavarapu. Solitary Plasmacytoma in Mandible with 7 Year Follow up Data. OHDM 2017;16(1): 1-4

[6]. B Sivapathasundharam, Arya Rajendran. Shafer's Textbook of Oral Pathology. 7th ed. Elsevier India ;2012. p. 124

[7]. Ibikunle A, Taiwo A, Braimah R, Adeyemi M. Solitary Plasmacytoma of the Mandible: Report of a Rare Case. International Biological and Biomedical Journal. 2018 Jan 10;4(1):67-71.

[8]. Sharma NK, Singh AK, Pandey A, Verma V. Solitary plasmacytoma of the mandible: A rare case report. Natl J Maxillofac Surg. 2015 Jan-Jun;6(1):76-9. Pubmed PMID: 26668458.

[9]. Dayisoylu EH, Ceneli O, Coskunoglu EZ. Solitary Plasmacytoma of the Mandible: An Uncommon Entity. Iran Red Crescent Med J. 2016 Feb 20;18(7):e22932. Pubmed PMID: 27651943.

[10]. Sharma RR, Kanwal P, Sehgal CM, Kapoor R, Rattan V, Mannja D, et al. Plasmacytoma of the Mandible. 